\title{
Quaderni
}

QUADERNI Communication, technologies, pouvoir

\section{Impératifs gestionnaires et phronesis médicale : esquisse sociologique d'un engagement éthique dans un grand hôpital parisien}

Managerial constraints and medical practical wisdom A sociological draft of an ethical commitment in a Parisian university hospital

\section{Frédéric Pierru}

\section{OpenEdition}

\section{Journals}

Édition électronique

URL : http://journals.openedition.org/quaderni/745

DOI : $10.4000 /$ quaderni.745

ISSN : 2105-2956

Éditeur

Les éditions de la Maison des sciences de l'Homme

Édition imprimée

Date de publication : 5 octobre 2013

Pagination : 67-82

\section{Référence électronique}

Frédéric Pierru, «Impératifs gestionnaires et phronesis médicale : esquisse sociologique d'un

engagement éthique dans un grand hôpital parisien », Quaderni [En ligne], 82 | Automne 2013, mis en ligne le 05 octobre 2015, consulté le 03 mai 2019. URL : http://journals.openedition.org/quaderni/745 ; DOI : 10.4000/quaderni.745 


\section{$D$ ossier}

\section{Impératifs} gestionnaires et phronesis médicale : esquisse sociologique

\section{d'un engagement éthique dans}

un grand

\section{hôpital parisien}

\author{
Frédéric \\ Pierru
}

CNRS - CERAPS
Pour les réformateurs de l'hôpital, l'affaire serait entendue : les rappels à l'éthique et à la déontologie que les professionnels de la médecine opposent à leurs préconisations ne seraient que le faux-nez de la défense d'un corporatisme étriqué et dépassé, de l'expression d'un archaïsme aux effets inflationnistes et aux conséquences délétères sur la qualité des soins. Pire, ils cacheraient, sous prétexte de nobles valeurs, la poursuite de «petits » intérêts économiques et la perpétuation d'arrangements quotidiens avec les « règles de l'art ». En cela, les réformateurs ont complètement fait leurs les critiques sociologiques du «premier» Eliot Freidson, celui de La profession médicale ${ }^{1}$, qui s'était employé à débusquer derrière la façade publique de l'éthique fondant les « privilèges » de la profession médicale, les ressorts des pratiques réelles, parfois peu reluisantes, de médecins abusant de leur autonomie et davantage soucieux de se protéger du regard de leurs patients que de mettre un terme aux mauvaises pratiques de leurs confrères. La critique gestionnaire de la profession médicale, bien qu'elle se revendique essentiellement de la légitimité du discours économique et de la « boîte à outils » du New Public Management, est l'héritière des critiques adressées à la médecine par les sciences sociales et la philosophie à compter du milieu des années 1960, sur fond de profondes transformations socioculturelles du rapport au corps, à la santé et à la médecine. Dès lors que l'éthique et la déontologie professionnelles ne suffisent pas à garantir l'économicité et la qualité des soins, il conviendrait de mettre en place un système d'incitations économiques, positives et négatives, adossé à des règles de bonne pratique élaborées par l'evidence based medicine. 
Le dialogue de sourds est presque total : là où les gestionnaires s'adressent d'abord aux intérêts économiques individuels des praticiens afin d'en infléchir les pratiques dans le sens de l'efficience, ces derniers dénoncent un «viol éthique » les obligeant à enfreindre les règles de l'art médical et à dispenser des soins de piètre qualité 2 . Comme l'a bien souligné Julia Evetts, ce conflit met aux prises deux acceptions concurrentes de ce qu'être professionnel veut dire. D'un côté, les gestionnaires en appellent à un "professionnalisme organisationnel » inspiré par le managérialisme, dans lequel la loyauté des médecins va d'abord à l'organisation (hospitalière) et les formes légales-rationnelles d'autorité, les procédures standardisées et les indicateurs de performance le disputent aux mécanismes de reddition de compte aux non-professionnels, à commencer par les patients ${ }^{3}$. De l'autre côté, les médecins défendent un «professionnalisme occupationnel» fondé sur la confiance des financeurs et des patients et dans lequel les formes d'autorité collégiale et le contrôle des pairs garantissent l'autonomie et le libre arbitre dans le travail quotidien et in fine la qualité des soins dispensés. Pour les gestionnaires, l'hôpital est une entreprise presque comme une autre, relevant des technologies de l'industrie ; pour les médecins, l'hôpital est une institution pluriséculaire. Les uns veulent instiller davantage d'intégration, de standardisation, de hiérarchie, de polyvalence, d'impératifs budgétaires de court terme; les autres valorisent l'hyper-spécialisation et la différenciation toujours plus poussée des services, la flexibilité de "l'ordre négocié », la coopération fondée sur le développement de savoirs partagés et collectivement validés, la poursuite de stratégies médicales inscrites sur les moyen et long termes...
Toutefois, dans la réalité, la ligne de front est plus heurtée et discontinue que cette opposition idéaletypique ne le suggère ${ }^{4}$. Ainsi, certains segments de la profession médicale sont plus prompts que d'autres, en raison notamment de la position occupée dans la division du travail et la hiérarchie médicales, à intégrer une partie des critiques gestionnaires tandis que certains spécialistes en gestion et en économie de la santé considèrent que " gérer la singularité à grande échelle $\|^{5}$ suppose de rompre avec le réflexe qui consisterait à transposer mécaniquement les technologies industrielles sur une activité de service travaillant sur de l'humain. Ces armistices pratiques signés par les acteurs de l'hôpital trouvent un écho dans la littérature sociologique. Ainsi, Eliot Freidson, pourfendeur du professionnalisme médical dans les années 1970, a peu à peu infléchi ses positions et plaidé pour un "renouveau $d u$ professionnalisme ", " troisième voie » entre le marché et l'État'. Si, en France, le tropisme critique de la sociologie interactionniste « classique » reste prégnant lorsqu'il s'agit d'étudier la médecine - ainsi que l'illustre la célébration des innovations introduites par les associations de patients -, la période récente a vu émerger des tentatives pour penser sociologiquement les impasses du managérialisme en santé. Des sociologues et des politistes, parfois en collaboration avec des praticiens, se sont efforcés de saisir «par le bas » les conséquences négatives sur les collectifs soignants, les pratiques médicales et la qualité des soins dispensés de l'entrée en gestion de l'hôpital, dans un contexte plus général de contestation professionnelle de l'offensive gestionnaire dans les services publics jusqu' alors dominés par des professions plus ou moins puissantes (magistrats, enseignants, travailleurs 
sociaux, etc.). La théorisation la plus aboutie de cette dialectique du managérialisme et du professionnalisme est, à ce jour, celle qui a été proposée par Florent Champy, avançant le concept de 《 professions à pratique prudentielle $»^{7}$. Même si certains ont vu dans cette dernière, un peu rapidement à notre sens, une résurgence de la sociologie fonctionnaliste contre laquelle s'est construite la sociologie interactionniste, il s'agit là d'une tentative originale pour conceptualiser, à partir des remarques d'Aristote sur la prudence mais aussi, plus sociologiquement, du « second Freidson » et des travaux de Andrew Abott, les menaces de diverses natures qui pèsent sur le travail professionnel, étant entendu que celui-ci présente des spécificités du point de vue de son contenu même : pratiquer la médecine, ce n'est pas, comme le prétendent certains sociologues interactionnistes, se livrer à l'activité de plombier. Pour F. Champy, l'autonomie délibérative de certaines professions devrait être préservée de certaines entraves en tant qu'elle garantirait la qualité des services par elles dispensés ${ }^{8}$. Pour autant, il ne s'agit pas là d'une posture « réactionnaire »: le sociologue a bien entendu pris acte des critiques adressées au professionnalisme traditionnel et il s'efforce donc de distinguer « bonne » et « mauvaise » autonomie.

Nous proposons dans cet article d'illustrer la pertinence du concept de « profession à pratique prudentielle » pour penser les conditions sociales de possibilité et de félicité, de l'engagement « éthique » de certains secteurs de la médecine en réaction à la montée en puissance des préoccupations et instruments gestionnaires à l'hôpital' Grâce à l'observation participante d'un groupe pluri-professionnel de « réflexion éthique » ini- tié conjointement par la praticienne responsable d'une Permanence d'accès aux soins de santé (PASS) et de la responsable du service social d'un CHU parisien ${ }^{10}$, nous mettrons d'abord en évidence les conditions sociales de possibilité de la réflexivité « éthique » : des effets de position et de disposition prédisposent certains professionnels à s'engager dans une réflexion sur les conséquences à tirer, du point de vue de l'éthique professionnelle, de la montée en puissance des impératifs gestionnaires sur les pratiques médicales dominantes. L'investissement de la cause, du registre et du discours de « l'éthique », telle qu'ils sont promus, depuis les années 1980 en France, par les « gardiens du corps $\rangle^{11}$, est la voie empruntée par ces professionnels occupant une position plutôt dominée dans le champ médical pour penser les injonctions paradoxales dans lesquels ils sont pris au quotidien, tout en convertissant leur stigmate médico-social en emblème « éthique $»^{12}$. En effet, le stigmate attaché à la pratique d'une médecine généraliste pratiquée auprès de populations étrangères confrontées à une grande précarité juridique et sociale, exercée dans l'un des temples de la biomédecine triomphante, est alors pleinement assumé et revendiqué, et devient même le mobile d'un engagement en faveur d'une conception plus « prudentielle » de l'éthique médicale posée en concurrente de la conception dominante selon laquelle tout doit être fait pour restaurer la santé du patient. Cependant, nous verrons que la réflexivité éthique se heurte rapidement à des limites politiques : certaines associations humanitaires rappellent à l'ordre éthique traditionnel ces entrepreneurs, au nom de l'égal accès de tous aux mêmes soins, quels qu'en soient le coût et la pertinence. Où l'on constate que, dans le cas présent, ce ne sont pas les médecins qui se mon- 
trent « corporatistes » et frileux, mais bien leur environnement institutionnel et politique.

\section{Les conditions sociales de possibilité d'un engagement éthique}

Le débat public tend à mettre en scène et à dramatiser l'opposition entre médecins hospitaliers et gestionnaires. Ce fut notamment le cas lors de l'élaboration de la loi « Hôpital, Patients, Santé, Territoires », qui fut à l'origine de la manifestation inédite de nombre d'hospitalo-universitaires parisiens de renom aux côtés des syndicats de personnels soignants et administratifs dans les rues de Paris au printemps $2009^{13}$. Nombre de médias s'amusèrent à cette occasion de voir défiler ensemble «mandarins de droite » et syndicalistes de la CGT notamment. Cette loi, qui affirmait l'autorité du chef d'établissement sur l'ensemble du corps médical dans le cadre d'une nouvelle « gouvernance » calée sur celle de l'entreprise, cristallisa le ressentiment de l'élite médicale à l'encontre d'un semble d'innovations gestionnaires introduites à compter du début des années 2000, au premier rang desquelles figure la fameuse « tarification à l'activité » (T2A) : montant en charge rapidement à partir de 2005, celle-ci fait dépendre le budget des établissements hospitaliers, en ce qui concerne la médecine, la chirurgie et l'obstétrique (MCO) du volume d'activité effectivement réalisé. En d'autres termes, les pouvoirs publics ne financent plus une structure (via le versement d'une dotation globale de fonctionnement) mais un service rendu, l'argent suivant les patients. Pour nombre de médecins, un tel mode de financement à l'activité est intrinsèquement pervers sur le plan éthique en tant qu'il incite à « faire du chiffre », à « soigner les indicateurs plutôt que les malades » et in fine à dégrader la qualité des soins de façon à rentrer dans les clous des tarifs fixés par le ministère. Tout comme les pôles médico-gestionnaires, visant à regrouper et se substituer aux traditionnels services, présentés par les réformateurs comme autant de "fiefs » inexpugnables, la T2A est en général dénoncée par les praticiens hospitaliers comme l'immixtion de la rationalité économique au cœur de l'activité de soin. Cette immixtion serait une constitutive d'une contamination de l'éthique médicale par des mobiles économiques considérés a priori comme illégitimes.

Néanmoins, comme toute réforme, celle de l'hôpital redistribue les chances, pour employer un terme wébérien, entre les groupes professionnels travaillant en son sein. Plus précisément, la logique gestionnaire est réfractée par la structure du champ médical dont l'autonomie, si elle tend à être remise en cause, n'en demeure pas moins une réalité. Certaines spécialités s'accommodent, voire tirent profit, des nouvelles règles de gestion. Ainsi, la T2A, parce qu'elle encapsule une définition de l'hôpital comme usine à soins techniques, valorise d'autant mieux l'activité que celle-ci est spécialisée, technique, programmable, standardisée, autrement dit qu'elle présente des affinités électives avec la logique industrielle. C'est, par exemple, le cas de la chirurgie orthopédique. À l'inverse, plus une activité médicale sollicite l'acte intellectuel aux dépens du recours à la technique, plus son succès dépend de la « compliance » du patient singulier, véritable co-producteur du soin, moins elle cadre avec la rationalité industrielle de la T2A. Ce n'est pas par hasard qu'un diabétologue et un psychiatre ont pris la tête de la fronde hospitalo-univer- 
sitaire contre les réformes hospitalières récentes. C'est encore plus vrai d'autres segments de la profession médicale, dont la vocation «médicosociale ", déjà peu valorisée et valorisante pour celles et ceux qui l'assument, se retrouvent encore plus marginalisée au sein de l'hôpital-usine à soins techniques et (hyper-)spécialisés ${ }^{14}$. Telle est la situation de ces interfaces entre la ville et l'hôpital que sont la médecine d'urgence ou la médecine exercée au sein des permanences d'accès aux soins de santé (PASS), qui régulent l'accès aux services spécialisés : il faut avant toute chose trier « bons » et « mauvais » malades, i.e. les patients «purs » et « impurs » selon les critères de jugement biomédical ${ }^{15}$. Ici, la gestion de flux et ses implications organisationnelles comptent autant sinon plus que la virtuosité clinique. La domination médicale, affirmée dans les services spécialisés, y cède du terrain au profit d'équipes semi-autonomes où les relations entre soignants, entre soignants et administratifs y sont plus horizontales. L'observateur est frappé par le caractère « détendu » des relations entre les différentes catégories de personnel. Last but not least, le financement de ces services déroge en tout ou en partie à la T2A : composite pour les urgences - forfaitaires et à l'activité -, il est complètement forfaitaire pour les PASS, qui relèvent des « missions d'intérêt général et d'aide à la contractualisation » (MIGAC), enveloppes budgétaires destinées à financer les activités de service public ne relevant pas de la T2A.

Située dans le centre hospitalo-universitaire Saint-Louis, au cœur d'un arrondissement populaire de la capitale, la consultation Verlaine est une PASS « dédiée » qui regroupe en un seul lieu des personnels spécialement affectés à la "prise en charge médico-sociale [devant] faciliter l'accès aux soins des personnes démunies au système hospitalier $»$ selon les termes du code de santé publique. Rattachés au service de médecine interne du CHU, ses effectifs sont modestes : sa responsable est un praticien hospitalier qui y consacre $60 \%$ de son temps ; elle est épaulée par onze vacations de praticien attaché, un interne et surtout par deux infirmières à temps plein et deux assistantes sociales. La présence renforcée des infirmières et des assistantes sociales au regard de la modestie des effectifs médicaux tient aux missions de filtre et de tri, mais aussi d'accompagnement et d'ouverture des droits sociaux éventuels des patients : les médecins ne reçoivent que les patients qui le nécessitent vraiment. Ceci étant dit, une telle unité occupe une position dominée sous nombre de rapports. Dominée, elle l'est d'abord en raison des stigmates attachés à sa « clientèle »: en l'occurrence des étrangers en situation souvent irrégulière, sans couverture sociale pour les deux tiers, et présentant souvent des problématiques médico-sociales complexes et lourdes (cancers, hémopathies, insuffisances rénales, VIH, etc.) du fait que l'hôpital Saint-Louis est réputé pour la cancérologie. Ces patients, dont le nombre est en force croissance depuis des années ( $+72 \%$ depuis 2006,5000 consultations annuelles en moyenne), sont adressés par de multiples acteurs du système de santé : associations humanitaires type Médecins du Monde, médecine générale de ville, centres de santé, urgences, consultations et services spécialisés de l'hôpital ${ }^{16}$. En tant qu'il s'y pratique une médecine généraliste auprès de patients « impurs » au regard de la logique biomédicale, un tel service est de facto stigmatisé dans un haut lieu de la médecine hospitalo-universitaire spécialisée. Même dans 
son service de rattachement, à savoir le service de médecine interne, spécialité médicale relativement dominée dans l'espace des spécialités médicales ${ }^{17}$, elle occupe une position subordonnée puisque la polyvalence revendiquée par sa responsable, spécialiste en rhumatologie, est en porte-à-faux avec la tendance des internistes de CHU à se présenter comme les spécialistes de maladies rares afin de redorer leur blason dans la hiérarchie des spécialités hospitalières. En un mot, prenant en charge le « sale boulot» dans le cadre d'une médecine « polyvalente » ou généraliste étroitement adossée au travail infirmier et social (il faut ouvrir les droits sociaux quand ils existent), contrainte de s'intéresser à des questions organisationnelles de gestion de flux, la médecine de PASS se retrouve assez éloignée, au sens symbolique, de la noblesse de la spécialisation biomédicale. Cette distance sociale et symbolique prédispose ses agents à porter un regard critique sur les pratiques d'une biomédecine hyperspécialisée et techniciste incitée de surcroît, par la T2A, à devenir productiviste ( « faire du chiffre $»)^{18}$. La responsable de la PASS est particulièrement bien placée pour constater que ses patients, qu'elle doit orienter vers la «bonne» spécialité, entrent assez difficilement dans les cases biomédicales étant donné l'intrication des problématiques sociales, médicales et parfois psychologiques. Du reste, en tant que spécialiste rhumatologue, discipline qui couvre un champ très large et varié, qui valorise l'acte intellectuel clinique et qui noue des interrelations étroites avec d'autres spécialités, ce médecin présente des propriétés en phase avec les exigences et les qualités requises par la consultation Verlaine qu'elle intègre progressivement à compter de 1996. Résumons, à ce stade, certaines des conditions sociales de possibilité d'un retour critique sur les pratiques biomédicales dominantes et l'éthique hippocratique qui leur est liée. Médecine des marges sociales, la médecine de PASS est en marge de l'excellence hospitalo-universitaire qu'elle côtoie cependant au quotidien, étant en position pour observer l'inadéquation de nombre de prises en charges spécialisées pour des situations médico-sociales complexes. Tout comme le service d'urgence, la consultation Verlaine est le lieu d'exercice d'une médecine hybride, mêlant médecine sociale empirique et administration de soins relativement sophistiqués, et consciente des limites des traitements médicaux pour des patients appelés à retrouver des conditions d'existence souvent précaires ${ }^{19}$. L'illusio biomédical et l'adhésion aux normes dominantes du champ médical y sont plus faibles. De plus, jouant le rôle d'interface ou de «plaque tournante » entre médecine générale (urgences, médecine humanitaire, médecine de ville) et les services hospitalo-universitaires spécialisés de pointe, elle occupe une position que l'on dira intermédiaire, particulièrement propice à l'accès à la pensée " pensante » prenant pour objet ici la doxa et le sens pratique de la biomédecine ${ }^{20}$. Dominée chez les dominants, la responsable de la consultation Verlaine est prédisposée à mener une réflexion critique sur les pratiques de ses confrères spécialistes plus prestigieux.

En 2006, l'inscription de deux situations particulièrement « aporétiques » sur cet horizon d'attente va être à l'origine de l'engagement de ce médecin dans une réflexion sur l'éthique clinique. L'une d'entre elles concerne Madame B., camerounaise ayant laissé ses deux enfants au pays. Souffrant d'un cancer métastatique d'abord traité 
au Cameroun, elle est venue en France, où elle est hébergée par une compatriote, dans l'espoir de guérir. Elle a été adressée à la consultation Verlaine par le service de cancérologie puisque, n'ayant pas de couverture sociale, il faut lui ouvrir d'éventuels droits à l'Aide Médicale d'État (AME) et lui dispenser, en attendant, le traitement prescrit par la cancérologue à titre gratuit. Or la responsable de la PASS est frappée par le fait que ce traitement est à la fois inutile et coûteux tout en obligeant la patiente à rester en France en situation de précarité et loin de sa famille. Particulièrement sensibilisée à la dimension économique des soins en raison de sa trajectoire sociale et professionnelle (elle a obtenu notamment un DEA de santé publique), s'affrontant aux fortes contraintes budgétaires qui pèsent sur son service - financé sur une enveloppe MIGAC insuffisante de l'avis général -, mais aussi à la nécessité de prendre en compte l'intrication des problématiques médicales, économiques, sociales, le médecin responsable de la PASS se montre critique à l'égard du choix de la cancérologue, focalisée sur la seule dimension technique et le principe hippocratique selon lequel tout ce qu'il est possible de faire doit être tenté pour la santé de la patiente. Ce réflexe interventionniste est d'ailleurs partagé par l'assistante sociale de la consultation dont l'éthos la porte à s'arranger avec les textes en vigueur pour ouvrir les droits sociaux nécessaires à la prise en charge médicale.

D’une façon générale, les personnels hospitaliers sont de plus en plus contraints de trancher euxmêmes ces douloureuses questions de rationnement que se refusent à aborder officiellement les pouvoirs publics, selon une logique de « politique du guichet » bien analysée par Alexis Spire ${ }^{21}$.
Le rationnement, ainsi ristourné à la discrétion des professionnels de terrain, est alors sérialisé, individualisé, « invisibilisé » et dépolitisé. Pour les réformateurs, la contrainte budgétaire constituerait une incitation externe à la mobilisation des gisements de productivité dormant sous l'épaisse couche bureaucratique et professionnelle ${ }^{22}$. L'enquête sociologique fait apparaître une tout autre réalité : la multiplication de «choix pathétiques » impliquant « des actions visant à allouer des ressources rares, qui conditionnent l'existence matérielle et sociale des personnes concernées et dont l'attribution se décide dans un contexte de mise en spectacle ou en discours de la souffrance $»^{23}$ alourdit la charge mentale du travail d'acteurs hospitaliers ayant le sentiment de n'être plus en mesure de dispenser des soins de qualité à tous. Une solution pour esquiver ce genre de choix tragiques réside dans le contournement de la législation, comme nous l'a confié la responsable du service social de l'hôpital ${ }^{24}$ : il s'agit alors de «monter des dossiers d'Aide Médicale d'État » coûte que coûte, en dépit du fait que les critères d'éligibilité ne sont pas remplis. Une autre voie consiste à expliciter ce qui, d'ordinaire, reste implicite en faisant l'objet d'arrangements informels entre personnels soignants et non-soignants concernés.

\section{La conversion du stigmate médico-social en emblème éthique}

Lieux de convergence et de cristallisation des tensions qui travaillent l'ensemble d'un système de santé soumis à une contrainte financière de plus en plus resserrée, des services comme les urgences ou les PASS sont bien placés pour prendre la pleine mesure de la « crise de l'hôpital ». Ainsi, 
la « dramaturgie de l'urgentiste », incarnée par les interpellations médiatiques d'un Patrick Pelloux, est au service de la revendication d'une meilleure reconnaissance matérielle et symbolique de cette spécialité déclassée et de l'attribution de moyens humains supplémentaires ${ }^{25}$. Il s'agit, dans cette perspective, de réclamer toujours plus de moyens sans questionner le paradigme biomédical dominant alors même que, «forts de leur expérience auprès d'usagers difficiles à classer selon le calibrage médical et critériologique », les urgentistes devraient, selon François Danet, «construire une clinique du "sujet global »qu'ils pourraient transmettre au reste du système de soins $»^{26}$. C'est précisément le sens de l'engagement « éthique » de la responsable de la consultation Verlaine, qui vise ainsi à la distinguer en montrant sa plusvalue par rapport au service d'urgence de l'hôpital Saint-Louis.

La multiplication des « choix pathétiques » que doivent opérer des soignants confrontés à l'afflux de migrants présentant des pathologies lourdes aggravées par une grande précarité socio-économique et juridique, dans un contexte de renforcement des contraintes budgétaires et de durcissement des politiques d'immigration invite, on l'a dit, à questionner les manières de faire ordinaires et les arrangements informels quotidiens avec la réglementation. Cette situation objective est une condition nécessaire de la réflexivité mais elle n'est pas suffisante. Il lui faut aussi s'exprimer selon des voies et dans des formes reconnues et légitimes, tant la sensibilité de l'enjeu du rationnement des soins est forte. Là où les urgentistes versent plutôt dans la dénonciation politique et médiatique de $l^{\prime}$ ' incompétence » et de $l^{\prime}$ « incurie » des décideurs politico-administratifs, la responsable de la consultation Verlaine préfère quant à elle « communiquer » sur la pertinence et l'apport des PASS dans la prise en charge des situations médico-sociales complexes. À cet égard, elle a largement contribué à la structuration d'un « collectif PASS » national destiné à faire mieux connaître du public et des responsables l'intérêt de ce type de structures dédiées à l'accueil des plus démunis. Ce militantisme relève de la stratégie de la divulgation identifiée par Goffman, laquelle vise à convertir le stigmate médico-social en emblème d'une médecine sobre centrée sur une prise en charge globale du patient.

L'engagement éthique est le point ultime de cette visée d'anoblissement. Dominique Memmi a bien identifié les propriétés et topiques de l'espace éthique qui se déploie dans les années $1980^{27}$ : lutte contre la pulsion d'emprise et l'agressivité des sciences biomédicales ; critique de l'inflation technologique et médicamenteuse ; souci constant de la « personne » envisagée dans sa globalité ; sens de la mesure, de la prudence, de la distance à l'urgence ; apologie des échanges pluridisciplinaires civilisés à rebours des parti-pris politiques, recherche du consensus par la délibération, etc. Le discours éthique des " gardiens du corps » est aujourd'hui légitime. L'éthique est une cause que peuvent endosser des soignants confrontés à des injonctions paradoxales. Ainsi Emmanuel Hirsch a fondé un «Espace Ethique » à l'Assistance Publique des Hôpitaux de Paris dont les locaux sont situés non loin de ceux de la consultation Verlaine, à l'hôpital Saint-Louis... Cette proximité géographique n'a pas été sans jouer un rôle dans l'engagement éthique du médecin responsable. Mais il faut aussi souligner combien la spécialité de médecine interne, dont l'identité 
est tiraillée entre l'apologie de la polyvalence, de la globalité et de la transversalité d'un côté, la conformité à la norme dominante d'hyperspécialisation de l'autre, incline à ce type de questionnement. L'avant dernier président du Conseil Consultatif National d'Ethique (CCNE), Didier Sicard, était d'ailleurs issu de ses rangs. Les affinités électives entre médecine interne et police éthique apparaissent ainsi clairement dans le Livre Blanc de la médecine interne de $2004^{28}$.

La responsable de la consultation et une cadre du service social très investie dans les problématiques de précarité se rapprochent donc de l'Espace Ethique pour finalement mettre en place, en 2007, un groupe pluri-professionnel de réflexion éloquemment baptisé « Équité face à l'accès aux soins dans le cadre des contraintes budgétaires actuelles ». Ouvert à tous les correspondants spécialistes de la consultation Verlaine, cherchant à associer médecins, infirmières, assistantes sociales, représentants de l'administration, il s'agit de réfléchir, une fois tous les deux mois, aux modalités concrètes de conciliation des principes fondamentaux de l'éthique tels qu'ils ont été explicités dans la littérature anglo-saxonne lorsque les soignants sont confrontés à des situations médico-sociales complexes. En effet, des pathologies lourdes associées à une grande précarité sociale et juridique rendent ardu l'établissement d'un compromis entre les principes de respect de l'autonomie de la personne, de bienfaisance (faire le bien certes, mais quel bien ?), de non-malfaisance (faut-il prescrire une chimiothérapie aux effets secondaires pénibles à une migrante isolée et sans ressources pour se nourrir ou se déplacer ?), de justice (justice ou équité ?). La conjonction de contraintes budgétaires tangibles (puisque la consultation Verlaine fonctionne avec une enveloppe budgétaire limitative) et de l'afflux de patients médicalement « lourds » et très précaires précipite ainsi une interrogation collective sur les coûteuses pratiques de prescription de spécialistes hospitaliers pour lesquels « tout doit être tenté techniquement pour sauver le patient» sans considération pour la situation socio-économique et le vécu de ce dernier. Ici, l'Éthique fournit à la fois les concepts (la formalisation des principes éthiques fondamentaux), la méthode (la délibération collégiale), la finalité (parvenir à un consensus sur les critères de la prise de décision médicale et extra-médicale) mais aussi la légitimité pour traiter explicitement et ouvertement de l'épineuse question de la gestion de la rareté et des « choix pathétiques », d'ordinaire esquivée ou résolue dans des marchandages informels entre soignants. Mais le succès n'est guère au rendezvous : les deux initiatrices se heurtent au relatif désintérêt de leurs correspondants spécialistes. Si un hématologue ou un cancérologue, poussés par la curiosité, viennent ponctuellement, ils ne s'investissent guère. La marginalité institutionnelle de la PASS joue à l'évidence ici à plein, tout comme l'emprise du paradigme biomédical dans les services spécialisés du CHU.

Pour autant, les deux entrepreneuses en éthique ne désarment pas et décident de poursuivre leur réflexion en y associant, à compter de 2009, une consultante en relation publique et en communication santé, se réclamant d'une compétence d'anthropologue, qui leur a été recommandée à l'hôpital Georges Pompidou ${ }^{29}$. Cette dernière leur propose de formaliser leurs échanges à l'aide d'une grille décomposant les dimensions médicales et non-médicales de la décision et en 
leur associant un score chiffré. Sont ainsi isolées trois dimensions principales : clinique bien sûr, mais aussi administrative et "sociologique ". Les items administratifs renvoient essentiellement aux dimensions juridique et économique de la décision (éligibilité à des droits sociaux, coût du traitement, etc.) tandis que les items sociologiques s'efforcent de cerner à la fois la situation sociale des patients - hébergement, soutien familial et amical en France - mais aussi certaines de leurs caractéristiques individuelles - projet de vie, motivation, capacité d'adaptation, « qualité de la relation avec les équipes ». Cette grille a été ensuite mise à l'épreuve de cas concrets (une soixantaine au total entre 2009 et 2012) dans le cadre de réunions de concertations pluri-disciplinaires médico-sociales et éthiques (RCP MSE), mises en place sur le modèle des RCP introduite par le premier plan cancer. La discussion associe la plupart du temps la médecin responsable de la consultation, le médecin spécialiste correspondant, un autre médecin de la même spécialité (pour un deuxième avis), la responsable du service social de l'hôpital, une infirmière, un représentant de l'administration de l'hôpital.

\section{Des entrepreneuses en éthique démunies quand la bise associative fut venue}

Il n'est pas possible ici de décrire plus précisément le processus d'élaboration de cette grille et la discussion des critères qui l'a scandée. Soulignons seulement l'originalité de la démarche : sollicitation du registre éthique tel qu'il a été défini en France à compter des années 1980 pour alléger la charge mentale induite par des choix pathétiques effectués sous de fortes contraintes budgétaires ; délibération collégiale ; intégration des dimensions clinique, médicale, sociale dans une grille décisionnelle formalisée et quantifiée. Cette expérience démontre avec éclat que les acteurs hospitaliers de terrain, contrairement à ce que pensent en général les réformateurs de l'hôpital, peuvent s'engager dans une réflexion sur les ressorts et les limites de leurs pratiques quotidiennes. Plus sociologiquement, cet engagement éthique permet d'objectiver les spécificités du raisonnement prudentiel tel que Florent Champy les a théorisées : confrontés à des cas singuliers et à des situations éminemment complexes et incertaines, donc échappant à toute maîtrise systématique, les enquêtés se sentent en l'espèce contraints d'expliciter ce qui fait le cœur de leur activité professionnelle mais qui reste d'ordinaire à l'état implicite : les différentes valeurs, potentiellement contradictoires, entrant en ligne de compte dans leur délibération conjecturelle ${ }^{30}$. Il s'agit pour eux de parvenir à un relatif consensus sur la hiérarchisation, pour une part irréductible, arbitraire des finalités de leur activité (via un dispositif de quantification et de pondération).

Cependant, le rappel à l'ordre de ces entrepreneuses en éthique ne va pas venir de la hiérarchie médicale ou de l'administration, mais de l'extérieur, en l'occurrence du mouvement associatif. Un collectif d'associations impliquées dans la défense et la promotion de la santé des étrangers (COMEDE, MDM, MSF, Act-Up) va avoir vent des réflexions menées à la consultation Verlaine et s'en émouvoir non seulement auprès des intéressés mais aussi et surtout auprès de la direction de l'hôpital, à la direction générale de l'AP-HP, au directeur général de l'organisation des soins du ministère de la Santé, au directeur général 
de l'agence régionale de santé d'Île-de-France. Les griefs sont multiples : discriminations dans l'accès aux soins de patients étrangers ; illégitimité/illégalité des critères administratifs et sociologiques de la grille (prise en considération du coût des soins, de l'accessibilité du traitement dans le pays d'origine, appréciation du projet de vie et des liens familiaux en France et dans le pays d'origine, etc.) ; atteinte au secret médical par des RCP associant médecins et non-médecins (infirmières, travailleurs sociaux, représentants de la direction); confusion entre médecine de contrôle et fonction de soignant, etc. Les associations en appellent ainsi au respect strict des textes juridiques (Convention européenne de sauvegarde des droits de l'Homme et des Libertés Fondamentales, code de la santé publique, circulaires administratives, code de déontologie médicale) pour mettre en cause l'engagement éthique des responsables de la PASS. Comme nous l'avons souligné, c'est la position à la fois dominée et intermédiaire de la PASS qui explique en grande partie l'émergence d'une réflexion critique originale sur les pratiques de prescription des services spécialisés. Mais cette position, en un sens, privilégiée «d'observatoire de l'accès aux soins et des pratiques médicales » est aussi l'envers d'une faiblesse institutionnelle. En effet, face à la mobilisation d'un groupe d'intérêt réputé influent, les maigres soutiens institutionnels tendent à faire défection.

Dans cette controverse, s'affrontent raisonnement prudentiel du médecin d'un côté, rationalité juridique des associations de l'autre. Pour le premier, l'application stricte des textes est non seulement impossible, étant donné la contrainte budgétaire, mais aussi contre-productive. Si, par exemple, les textes régissant l'accès à l'AME étaient strictement appliqués par les assistantes sociales, alors nombre de patients se présentant à la consultation ne seraient pas éligibles puisqu'ils sont venus en France dans l'unique intention d'accéder à des traitements médicaux non disponibles dans leur pays d'origine. Plus gravement, un respect mécanique de la réglementation conduirait à des absurdités médicales et humaines : comment est-il possible de prescrire une chimiothérapie lourde à un patient étranger vivant isolé dans la rue, dans la clandestinité, et n'ayant pas assez d'argent pour se nourrir, voire acheter un simple ticket de métro pour se rendre à l'hôpital ? Dès lors, pour ce médecin, il convient parfois de s'écarter de la règle pour mieux hiérarchiser les finalités de l'intervention et de trouver, en considérant la complexité médico-sociale, la «moins mauvaise des solutions possibles » pour le patient. Pour les associations, au contraire, les principes juridiques ne se négocient pas au cas par cas : l'égalité d'accès aux soins implique de dispenser aux étrangers et aux assurés sociaux les mêmes traitements. Il ne peut y avoir exception à la règle sauf à discriminer des populations très vulnérables.

Cette controverse est aussi de nature politique et, de fait, elle permet d'identifier les limites du registre éthique. Les professionnels de la consultation Verlaine, pris par l'urgence pratique, font, en effet, avec le cadre budgétaire et réglementaire tel qu'il existe. Ils cherchent à l'accommoder et à s'en accommoder en tentant de trouver une méthode pratique et consensuelle pour gérer la contrainte budgétaire et, surtout, pour s'adapter aux situations humainement douloureuses des migrants. Comme l'ont souligné Dominique 
Memmi $^{31}$ mais aussi Pierre-André Taguieff ${ }^{32}$, l'éthique vise, par la discussion et le compromis, à élaborer " un monde commun » permettant de civiliser les rapports sociaux de domination, d'apaiser les esprits, d'éteindre les polémiques partisanes. Dans l'éthique, la « guerre des Dieux » ne trouve guère sa place. Bref, l'éthique tend à dépolitiser le monde social. Certes, la responsable de la PASS est consciente des facteurs géopolitiques des situations singulières qu'elle affronte quotidiennement. Mais elle doit en prendre acte. $\mathrm{Au}$ contraire, les porte-parole associatifs sont libérés des impératifs de la logique pratique. Ils peuvent, ainsi, s'appuyer sur les textes pour scandaliser et politiser un rationnement « éthique » opérant au ras du sol. Le dispositif éthique de la consultation Verlaine sert alors, au moins partiellement, de prétexte pour attirer l'attention sur la condition inhumaine faite aux migrants, sur l'inégalité et les discriminations dont ils sont victimes. Convoqués dans cette controverse, les gestionnaires tirent profit de leur double jeu habituel : poussant les soignants, par le déploiement d'instruments $a d$ hoc à faire les choix pathétiques (ou tragiques) qu'ils se refusent à endosser publiquement, ils peuvent toujours, lorsque la situation est construite en scandale public par des acteurs extérieurs (associations, journalistes, etc.), se présenter comme les gardiens de la Loi généreuse et républicaine pour mieux blâmer les professionnels pour leurs défaillances « éthiques ». « Pile je gagne, face tu perds » : il serait étonnant, dans ces conditions, que l'incompréhension entre professionnels et gestionnaires ne s'aggravât pas.

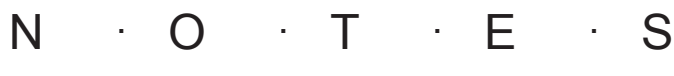

1. Freidson (Eliot), La profession médicale, Paris, Payot, 1984.

2. Mas (Bertrand) et al. (dir.), L'hôpital en réanimation, Bellecombe-en-Bauges, Éditions du Croquant, 2011 ; Collectif Docteurs blouses, «L'hôpital malade de l' « efficience » », Revue du MAUSS, n 41, 2013, pp. 53-75.

3. Evetts (Julia), « Trust and Professionalism: Challenges and Occupational Changes », Current Sociology, $\mathrm{n}^{\circ}$ 4, vol. 54, 2006, pp. 515-531.

4. Belorgey (Nicolas), L'hôpital sous pression. Enquête sur le nouveau management public, Paris, La Découverte, 2010.

5. Minvielle (Étienne), « Gérer la singularité à grande échelle », Revue française de gestion, $\mathrm{n}^{\circ} 109,1996$, pp. 114-125.

6. Freidson (Eliot), Professionalism Reborn: Theory, Prophecy and Policy, Cambridge, Polity Pres, 1994 ; Freidson (Eliot), Professionalism: The Third Logic, London, Polity Press, 2001.

7. Bezès (Philippe) et al., « New Public Management et les professions dans l'État : au-delà des oppositions, quelles recompositions ? ", Sociologie du travail, $\mathrm{n}^{\circ} 53,2011$, pp. 293-348.

8. Champy (Florent), La sociologie des professions, Paris, PUF, 2012.

9. Champy (Florent), Israël (Liora), « Professions et engagement public », Sociétés contemporaines, ${ }^{\circ} 1$, 2009, pp. 7-19.

10. Nous avons été associés aux réflexions de ce groupe à compter de 2009, après avoir été sollicité en tant que sociologue par son initiatrice. Depuis lors, nous échangeons régulièrement et avons été associés à plusieurs manifestations (colloque, séminaires) organisées par le médecin responsable de la PASS. Je 
remercie infiniment celle-ci pour cette opportunité et pour son aide précieuse et sa diligence à me fournir les documents en sa possession. Tous les extraits d'entretiens sont issus de l'enquête de l'auteur.

11. Memmi (Dominique), Les gardiens du corps. Dix ans de magistère bioéthique, Paris, Éditions de l'EHESS, 1996.

12. Goffman (Erving), Stigmates. Les usages sociaux des handicaps, Paris, Minuit, 1975.

13. Pierru (Frédéric), « Les mandarins à l'assaut de l'usine à soins. Bureaucratisation néolibérale de l'hôpital français et mobilisation de l'élite hospitalo-universitaire », dans Hibou (Béatrice) (dir.), La bureaucratisation néolibérale, Paris, La Découverte, 2013, pp. 203-230.

14. Mossé (Philippe), Le lit de Procuste. L'hôpital : impératifs économiques et missions sociales, Ramonville, Erès, 1997 ; Parizot (Isabelle), Soigner les exclus, Paris, PUF, 2003.

15. Dodier (Nicolas), Camus (Agnès), « L'hospitalité de l'hôpital. L'accueil et le tri des patients aux urgences médicales ", Annales ESC, n 65 , vol. 65, 1997, pp. 109-119.

16. Revue humanitaire, «Dossier : Krach sanitaire : la crise, à quel prix », n 30, décembre 2011.

17. Le recrutement social de la spécialité atteste de cette domination relative : occupant une position intermédiaire sous le rapport de la féminisation, elle recrute, comparativement à d'autres spécialités comme la chirurgie, la cardiologie ou même la rhumatologie, moins d'agents issus des catégories sociales supérieures. Elle constitue souvent l'un des derniers choix des admis au concours de l'internat. Jaisson (Marie), « La mort aurait-elle mauvais genre? ", Actes de la recherche en sciences sociales, $\mathrm{n}^{\circ} 143,2002$, pp. 44-52. 18. Plus généralement, on soulignera que plus une " spécialité » médicale revêt une vocation généraliste et sollicite l'acte intellectuel (versus l'acte technique) moins elle est valorisée à tous les sens du terme - monétaire et symbolique - dans le système de santé français. C'est donc préférentiellement dans ces secteurs de la médecine qu'émergent les mobilisations en faveur de la rénovation " éthique » de la pratique médicale. L'on pourrait citer l'exemple du Syndicat de la médecine générale (SMG), créé en 1975 par des médecins généralistes exerçant auprès de populations défavorisées, aux dispositions politiques marquées, qui édite la revue Pratiques. Les Cahiers de la médecine utopique.

19. Peneff (Jean), L'hôpital en urgence, Paris, Métailié, 1996.

20. Par analogie avec les analyses de Mauger (Gérard), «Sens pratique et conditions sociales de possibilité de la pensée « pensante »", Cité, n 38, 2009, p. 69.

21. Spire (Alexis), Accueillir ou reconduire. Enquête sur les guichets de l'immigration, Paris, Editions Raisons d'Agir, 2008.

22. Moisdon (Jean-Claude), « Le paradoxe de la boîte noire. Réformes hospitalières et organisation », Droit et société, $\mathrm{n}^{\circ}$ 80, 2012, pp. 91-115.

23. Fassin (Didier), « Le corps exposé. Essai d'économie morale de l'illégitimité », dans Fassin (Didier), Memmi (Dominique) (dir.), Le gouvernement des corps, Paris, Editions de l'EHESS, 2004, p. 253.

24. Jusot (Florence) et al., «Inégalités de santé liées à l'immigration en France. Effets des conditions de vie ou sélection à la migration », Revue économique, $\mathrm{n}^{\circ} 2$, vol. 60, 2009, pp. 385-411.

25. Danet (François), « La dramaturgie de l'urgentiste », Esprit, novembre 2006, p. 12.

26. Ibid.

27. Memmi (Dominique), Les gardiens du corps, op. cit.

28. Collège national de médecine interne, La médecine interne en France, 2004, pp. 26-27.

29. La responsable de la consultation décide parallè- 
lement de mener un master «Éthique, science, santé \& société ».

30. Champy (Florent), La sociologie des professions, op. cit., pp. 80-94.

31. Memmi (Dominique), Faire vivre et laisser mourir. Le gouvernement contemporain de la naissance et de la mort, Paris, La Découverte, 2003.

32. Taguieff (Pierre-André), La bioéthique ou le juste milieu : une quête de sens à l'âge du nihilisme technicien, Paris, Fayard, 2007.

\section{$R \cdot E ́ \cdot S \cdot U \cdot M \cdot E ́$}

Le sens commun comme les professionnels de santé tendent à dramatiser l'opposition entre rationalité gestionnaire d'un côté, éthique professionnelle de l'autre. Dans cette vision, les contraintes budgétaires et le déploiement des instruments de gestion seraient autant de périls pour les valeurs dont les soignants seraient les porteurs. Une enquête menée dans un service destiné à accueillir les populations les plus précarisées au sein d'un hôpital hospitalo-universitaire parisien montre, au contraire, que certains segments de la profession médicale sont particulièrement prédisposés non seulement à opérer un retour réflexif sur les pratiques biomédicales dominantes, mais aussi à retraduire les exigences gestionnaires en impératif éthique renouvelé. Ainsi, la cause éthique, institutionnalisée en France à compter du milieu des années 1980, est disponible pour des stratégies d'anoblissement de pratiques soignantes non seulement très contraintes économiquement mais aussi relativement stigmatisées dans le monde hospitalo-universitaire. Cependant, le paradoxe veut que ces entrepreneurs en éthique ne soient guère soutenus par les pouvoirs publics dès lors que des associations de défense des « usagers » se mobilisent pour dénoncer ce qu'elles considèrent constituer un rationnement illégitime de l'accès aux soins de pointe.

\footnotetext{
Abstract

Managerial constraints and medical practical wisdom A sociological draft of an ethical commitment in a Parisian university hospital

Common sense as health professionals tend to dramatize the conflict between managerial rationality on one side, professional ethics on the other. According to this point of view, budgetary constraints and implementa-
} 
tion of new managerial tools are many dangers to the values that health professionals cherish. A sociological survey of a service to treat the most vulnerable populations in a Parisian university hospital shows, however, that some segments of the medical profession are particularly susceptible not only to make a reflexive critique of dominant biomedical practices but also to retranslate economic constraints in a new ethical imperative. Thus, the ethical discourse, institutionalized in France in the mid-1980s, can be strategically used to enhance medical practices that are not only subjects to severe economic constraints but that are also relatively stigmatized in the biomedical world. However, the paradox is that these moral entrepreneurs are hardly supported by public officials when advocacy groups of patients and "users" are mobilizing to denounce what they consider to be an illegitimate rationing access to advanced care. 
